\title{
Nomogram Model for Predicting Early Onset of Chronic Kidney Disease Using Color Doppler Region of Interest Technique
}

\section{Liang Zhang}

The Affiliated Hospital of Qingdao University

Kuishuai Xu ( $\nabla 1213218702 @ q q . c o m$ )

The Affiliated Hospital of Qingdao University

\section{Leiyuan $\mathrm{Ma}$}

The Affiliated Hospital of Qingdao University

Changjie Shao

The Affiliated Hospital of Qingdao University

\section{Research}

Keywords: Color Doppler, Region of interest, Chronic kidney disease, Nomogram

Posted Date: November 11th, 2021

DOI: https://doi.org/10.21203/rs.3.rs-989353/v1

License: @ (i) This work is licensed under a Creative Commons Attribution 4.0 International License. Read Full License 


\section{Abstract}

Purpose: The risk factors of chronic kidney disease were analyzed by using the region of interest quantitative technology of color doppler combined with QLab software, and a Nomogram was established to conduct an individualized assessment of patients with chronic kidney disease.

Methods: A total of 500 patients with chronic kidney disease diagnosed in our hospital from June 2019 to March 2021 were selected as the chronic kidney disease group, and 300 healthy patients during the same period were selected as the control group. Univariate analysis was performed on the test indexes(Fasting blood glucose, total cholesterol, triglyceride, urea nitrogen , creatinine, uric acid, albumin, red blood cell count, glomerular filtration rate, urinary protein) and the vascularity index, flow index, and vascularization flow index measured by the color doppler region of interest quantitative technique. The above meaningful indicators were included in the Logistics regression analysis to obtain the independent risk factors of early chronic kidney disease. The independent risk factors were imported into R software to draw a nomogram model for predicting early chronic kidney disease and evaluate the model.

Results: Single factor analysis results suggest age, hypertension, diabetes, hyperlipidemia, disease of heart head blood-vessel, body mass index, vascularity index, flow index, and vascularization flow index, fasting blood sugar, triglyceride, total cholesterol, urea nitrogen, creatinine, uric acid, glomerular filtration rate differences statistically significant $(P<0.05)$, while gender, renal length to diameter, the thickness of the cortex, Resistance index and peak systolic velocity and albumin difference has no statistical significance $(P>0.05)$. Logistics regression analysis showed that hypertension, diabetes, flow index, and vascularization flow index, urea nitrogen and albumin were independent risk factors for the early occurrence of chronic kidney disease. The $\mathrm{C}$ index of this nomogram using independent risk factors is 0.896 (95\% Cl: $0.862-0.930)$, which indicates that the nomogram has good discriminant power. The receiver operating curve of the histograph was AUC(area under the curve) 0.884 (95\% Cl: $0.860-0.908$ ), with the optimal threshold of 0.663 , specificity of $88.7 \%$, sensitivity of $78.0 \%$, accuracy of $82.0 \%$, and positive predictive value of $91.9 \%$. The ROC(receiver operator characteristic curve) of urea nitrogen, albumin, flow index, and vascularization flow index were evaluated. The results indicated that the best cutoff value of urea nitrogen was $5.9 \mathrm{mmol} / \mathrm{L}$, flow index was 14.67 , vascularization flow index was 4.6, and albumin was $40.26 \mathrm{~g} / \mathrm{L}$.

Conclusion: In the prediction of chronic kidney disease I-II stage, the quantitative technique of color Doppler region of interest has certain diagnostic value. The model established in this study has good discriminative power and can be applied to clinical practice, giving certain indicative significance.

\section{Introduction}

In China, the prevalence rate of Chronic kidney disease (CKD) is increasing year by year, which has reached $11 \%$ at present [1-3]. The structure or function of the kidney is affected by pathological changes in renal tissue caused by various causes. With the decline of renal function, it gradually develops to end- 
stage renal disease, and then causes a series of complications and even death [4-6]. For CKD, early screening and diagnosis is undoubtedly an effective way to reduce mortality. Currently, the diagnosis of chronic kidney disease is mainly based on changes in glomerular filtration rate, endogenous creatinine clearance, and creatinine value, or on renal damage indicators in blood and urine, "gold standard" pathological results, and imaging findings [7-8]. In this study, the risk factors and laboratory indicators of CKD were analyzed using the region of interest quantitative technology of color Doppler with QLAB system, and a Nomogram model was established to provide an objective and individualized assessment of CKD patients.

\section{Materials And Methods}

\section{General information}

500 patients with CKD diagnosed in our hospital from June 2019 to March 2021 were selected as subjects, including 259 males and 241 females, with an average age of $48.2 \pm 15.0$ years. Inclusion criteria :(1) Conformed to the stage I-II diagnostic criteria of CKD [9]; (2) No other renal diseases; (3) No history of malignant tumor; (4) Complete clinical data and complete imaging examinations. A total of 300 healthy subjects were selected as control group, including 136 males and 164 females, with an average age of $36.8 \pm 19.8$ years.

\section{Instruments and methods}

Philips EPIQ7(Philips Ultrasound, Inc) was used as the ultrasonic diagnostic instrument, the frequency was set at $3.5 \mathrm{MHz}$, and the probe was $\mathrm{C} 5-1$. The examination was performed by the same physician. As instructed, the patient lies on the examination bed in a prone position. The patient is required to fully expose the lower back while connecting the ECG monitor. First, gray scale ultrasound scanning was performed on the subjects, and the standard section of the long axis of the kidney was selected. The echo of renal parenchyma, cortex and medulla was observed on the section plane. The length diameter and cortical thickness of the kidney were measured and recorded. At this point, ask the patient to hold his or her breath. The physician turns on color Doppler conditions to select the middle and lower poles of the kidney. After image stabilization, the peak systolic velocity (PSV) and vascular resistance index (RI) of the interlobar arteries were measured and recorded, and the color hemodynamics of 3 cycles were stored.

The stored hemodynamics were analyzed using QLAB 10 software(Philips). The region of interest (ROI) was set to be between the renal capsule and the renal vertebral body, with the adjacent arcuate artery as the side. Automatically track blood flow in ROI and analyze vascularity index ( $\mathrm{VI})$, flow index (FI), and vascu1 flow index $(\mathrm{VFI})$. The average values of $\mathrm{VI}, \mathrm{FI}$ and $\mathrm{VFI}$ for 3 periods were recorded. As is shown in the figure1.

\section{Laboratory indicators}


Blood glucose, total cholesterol, triglyceride, urea nitrogen (BUN), creatinine, uric acid, albumin, red blood cell count, glomerular filtration rate, and urine protein were recorded in all subjects.

\section{Statistical Methods}

SPSS V26.0 software(BDSC Cat\# 7008, RRID:BDSC_7008) was used for statistical analysis. All enumeration data were used for univariate analysis by $\chi 2$ test, while measurement data were used for univariate analysis by independent sample $t$ test and represented by $\mathrm{x} \pm \mathrm{s}$. Positive indicators with statistically significant differences after univariate analysis were included in Logistic regression analysis to evaluate independent risk factors. The above independent risk factors were imported into $R$ (RayBiotech Cat\# DS-MB-01199, RRID:AB_853316) 4.0.4 software, and the outcome was whether the patients had early CKD. Through the above steps, nomogram is constructed that can predict the risk of early CKD.

Draw a nomogram ROC, and the area under the curve (AUC) represents the discriminant power of this nomogram. (AUC=0.5 indicates poor discrimination ability, and $A U C=1.0$ indicates good discrimination ability of the histogram). When the reference index is greater than the cutoff value, there is a risk of CKD. Conversely, when the reference index is less than the cutoff value, there is no or lesser risk of CKD.The Nomogram was repeated for 1000 times using Bootstrap method for internal verification. Draw a Nomogram calibration curve to evaluate the correlation between the predicted CKD probability and the observed CKD results. Decision Curve Analysis (DCA) was drawn for evaluation. The obtained CIndex was used for difference differentiation assessment. All the above values were set as $\mathrm{P}<0.05$ to be statistically significant.

\section{Analysis}

In this study, we drew a calibration curve to verify the accuracy of the Nomogram. In this study, we tried to expand the sample size of the experimental group.

\section{Results}

\section{Baseline data}

Univariate analysis was performed on baseline data of 500 patients in the CKD group and 300 subjects in the control group. The results indicated that the differences in age, hypertension, diabetes, hyperlipidemia, cardiovascular and cerebrovascular diseases, $\mathrm{BMI}, \mathrm{VI}, \mathrm{FI}, \mathrm{VFI}$, fasting blood glucose, triglyceride, total cholesterol, BUN, creatinine, uric acid and glomerular filtration rate were statistically significant $(P<0.05)$. There were no significant differences in sex, renal length diameter, cortical thickness, RI, PSV and albumin $(P>0.05)$. Assign the data before analysis: male $=1$, female $=0$; Age 0-30=1, 30-60=2, 60-100=3;

Hypertension: positive $=1$, negative $=0$; Diabetes mellitus: positive $=1$, negative $=0$; Cardiovascular and cerebrovascular diseases: positive $=1$, negative $=0$; Hyperlipidemia: positive $=1$, negative $=0$; $\mathrm{BMl}$ : $\geq 24 \mathrm{~kg} / \mathrm{m}^{2}=1,<24 \mathrm{~kg} / \mathrm{m}^{2}=0$. (Table 1) 
Table 1

Univariate analysis of clinical data of the two groups

\begin{tabular}{|c|c|c|c|c|}
\hline projects & $\begin{array}{l}\text { CKD group } \\
(n=500)\end{array}$ & $\begin{array}{l}\text { control } \\
\text { group } \\
(n=300)\end{array}$ & $x^{2} / t$ & $P$ \\
\hline \multicolumn{5}{|l|}{ gender } \\
\hline male & 259 & 136 & 3.137 & 0.077 \\
\hline female & 241 & 164 & & \\
\hline \multicolumn{5}{|l|}{ Age } \\
\hline$<30$ & 41 & 85 & 57.471 & $<0.001$ \\
\hline $30-60$ & 326 & 149 & & \\
\hline$>60$ & 133 & 66 & & \\
\hline \multicolumn{5}{|l|}{ Hypertension } \\
\hline Yes & 234 & 33 & 142.658 & $<0.001$ \\
\hline No & 266 & 267 & & \\
\hline \multicolumn{5}{|l|}{ Diabetes mellitus } \\
\hline Yes & 185 & 18 & 95.156 & $<0.001$ \\
\hline No & 315 & 282 & & \\
\hline \multicolumn{5}{|c|}{$\begin{array}{l}\text { Cardiovascular and cerebrovascular } \\
\text { diseases }\end{array}$} \\
\hline Yes & 20 & 4 & 4.582 & 0.032 \\
\hline No & 480 & 296 & & \\
\hline \multicolumn{5}{|l|}{ Hyperlipidemia } \\
\hline Yes & 211 & 91 & 56.723 & $<0.001$ \\
\hline No & 289 & 209 & & \\
\hline \multicolumn{5}{|l|}{ BMI } \\
\hline$\geq 24 \mathrm{~kg} / \mathrm{m}^{2}$ & 181 & 133 & 28.910 & $<0.001$ \\
\hline$<24 \mathrm{~kg} / \mathrm{m}^{2}$ & 319 & 167 & & \\
\hline $\begin{array}{l}\text { Course of the disease } \\
\text { (months) }\end{array}$ & $18.4 \pm 39.6$ & - & - & - \\
\hline
\end{tabular}




\begin{tabular}{|c|c|c|c|c|}
\hline projects & $\begin{array}{l}\text { CKD group } \\
(n=500)\end{array}$ & $\begin{array}{l}\text { control } \\
\text { group } \\
(n=300)\end{array}$ & $x^{2} / t$ & $P$ \\
\hline $\mathrm{VI}$ & $29.9 \pm 10.0$ & $35.4 \pm 11.1$ & 7.281 & $<0.001$ \\
\hline $\mathrm{FI}$ & $14.8 \pm 2.2$ & $16.3 \pm 2.0$ & 10.190 & $<0.001$ \\
\hline VFI & $4.2 \pm 1.8$ & $5.8 \pm 1.7$ & 13.135 & $<0.001$ \\
\hline $\begin{array}{l}\text { The length of the kidney } \\
(\mathrm{cm})\end{array}$ & $9.7 \pm 2.2$ & $10.1 \pm 2.2$ & 4.419 & 0.216 \\
\hline Cortical thickness(cm) & $2.1 \pm 0.2$ & $2.2 \pm 0.3$ & 5.432 & 0.318 \\
\hline RI & $0.62 \pm 0.06$ & $0.61 \pm 0.06$ & 3.274 & 0.223 \\
\hline $\mathrm{PSV}(\mathrm{cm} / \mathrm{s})$ & $26.5 \pm 5.9$ & $27.5 \pm 5.8$ & 2.312 & 0.021 \\
\hline $\begin{array}{l}\text { Fasting plasma glucose } \\
(\mathrm{mmol} / \mathrm{L})\end{array}$ & $5.1 \pm 1.1$ & $4.8 \pm 0.5$ & 4.527 & $<0.001$ \\
\hline $\begin{array}{l}\text { Triglycerides } \\
(\mathrm{mmol} / \mathrm{L})\end{array}$ & $1.8 \pm 1.4$ & $1.4 \pm 0.7$ & 5.395 & $<0.001$ \\
\hline $\begin{array}{l}\text { Total cholesterol } \\
(\mathrm{mmol} / \mathrm{L})\end{array}$ & $6.2 \pm 2.3$ & $5.6 \pm 2.0$ & 4.016 & $<0.001$ \\
\hline BUN (mmol/L) & $6.7 \pm 3.1$ & $5.1 \pm 1.8$ & 9.131 & $<0.001$ \\
\hline Creatinine(umol/L) & $103.1 \pm 102.2$ & $46.9 \pm 15.4$ & 9.430 & $<0.001$ \\
\hline Uric acid (umol/L) & $351.0 \pm 113.9$ & $321.2 \pm 88.8$ & 3.980 & $<0.001$ \\
\hline $\operatorname{Albumin}(\mathrm{g} / \mathrm{L})$ & $30.6 \pm 8.5$ & $33.2 \pm 8.5$ & 4.105 & $<0.001$ \\
\hline Urinary erythrocyte count(/ul) & $263.5 \pm 1094.4$ & - & - & - \\
\hline eGFR(ml/min) & $100.1 \pm 16.6$ & $129.3 \pm 7.3$ & 15.359 & $<0.001$ \\
\hline Urine protein $(\mathrm{mg} / \mathrm{L})$ & $2234.8 \pm 2519.3$ & - & - & - \\
\hline
\end{tabular}

\section{Logistics regression analysis of the risk of chronic kidney disease}

The incidence of early chronic kidney disease was set as the dependent variable. Significant indicators from baseline data (age, hypertension, diabetes, cardiovascular and cerebrovascular diseases, hyperlipidemia, BMI, VI, FI, VFI, PSV, fasting blood glucose, triglyceride, total cholesterol, BUN, uric acid, 
and albumin) were included in Logistic regression analysis. The results showed that hypertension, diabetes, FI, VFI, BUN and albumin were independent risk factors for the early occurrence of chronic renal disease. (Table 2)

Table 2

Results of logistic regression analysis

\begin{tabular}{|c|c|c|c|c|c|c|}
\hline Related factors & $\begin{array}{l}\text { Regression } \\
\text { coefficient }\end{array}$ & $\begin{array}{l}\text { Standard } \\
\text { error }\end{array}$ & Wald & OR & $95 \% \mathrm{Cl}$ & $P$ \\
\hline Age & 0.004 & 0.005 & 0.666 & 1.004 & $\begin{array}{l}0.994- \\
1.015\end{array}$ & 0.415 \\
\hline Hypertension & -1.656 & 0.243 & 46.536 & 0.191 & $\begin{array}{l}0.119- \\
0.307\end{array}$ & $<0.001$ * \\
\hline Hyperlipidemia & -0.235 & 0.219 & 1.149 & 0.790 & $\begin{array}{l}0.514- \\
1.215\end{array}$ & 0.284 \\
\hline Diabetes & -1.683 & 0.304 & 30.690 & 0.186 & $\begin{array}{l}0.102- \\
0.337\end{array}$ & $<0.001$ * \\
\hline $\begin{array}{l}\text { Cardio-cerebrovascular } \\
\text { disease }\end{array}$ & -1.153 & 0.724 & 2.531 & 0.316 & $\begin{array}{l}0.076- \\
1.307\end{array}$ & 0.112 \\
\hline BMI & 0.134 & 0.211 & 0.407 & 1.144 & $\begin{array}{l}0.757- \\
1.729\end{array}$ & 0.524 \\
\hline VI & -0.016 & 0.010 & 2.575 & 0.109 & $\begin{array}{l}0.965- \\
1.004\end{array}$ & 0.984 \\
\hline $\mathrm{FI}$ & -0.281 & 0.050 & 31.965 & 0.755 & $\begin{array}{l}0.685- \\
0.832\end{array}$ & $<0.001^{*}$ \\
\hline VFI & -0.395 & 0.060 & 42.581 & 0.674 & $\begin{array}{l}0.599- \\
0.759\end{array}$ & $<0.001^{*}$ \\
\hline PSV & -0.350 & 0.018 & 3.723 & 0.966 & $\begin{array}{l}0.932- \\
1.001\end{array}$ & 0.054 \\
\hline Fasting plasma glucose & 0.120 & 0.134 & 0.801 & 1.128 & $\begin{array}{l}0.867- \\
1.468\end{array}$ & 0.371 \\
\hline Triglycerides & 0.161 & 0.126 & 1.622 & 1.175 & $\begin{array}{l}0.917- \\
1.505\end{array}$ & 0.203 \\
\hline Total cholesterol & 0.071 & 0.052 & 1.870 & 1.073 & $\begin{array}{l}0.970- \\
1.188\end{array}$ & 0.170 \\
\hline BUN & 0.170 & 0.050 & 11.570 & 1.186 & $\begin{array}{l}1.075- \\
1.308\end{array}$ & $0.001^{*}$ \\
\hline Uric acid & 0.000 & 0.001 & 0.053 & 1.000 & $\begin{array}{l}0.998- \\
1.002\end{array}$ & 0.819 \\
\hline Albumin & -0.026 & 0.012 & 4.487 & 0.974 & $\begin{array}{l}0.951- \\
0.998\end{array}$ & $0.034^{\star}$ \\
\hline
\end{tabular}




\section{Establishment of a Nomogram to predict the risk of early chronic kidney disease}

The independent risk factors (hypertension, diabetes, FI, VFI, BUN, and albumin) for the prediction of CKD were imported into $\mathrm{R}$ software according to Logistic regression analysis results, and the Nomogram model was established. The prediction model is as follows: logit(CKD) $=6.19247+1.80654{ }^{\star} B P$ $-0.28469 * \mathrm{FI}+1.82764 * \mathrm{DM}+0.19816 * \mathrm{BUN}-0.40586 * \mathrm{VFI}-0.03823 * \mathrm{ALB}$. The procedure for using Nomogram to predict the risk of early onset of CKD is as follows: Find the score of each predictor on the corresponding vertical line in the Nomogram. Calculate the sum of the six indicators. The probability of risk is the value of CKD on the vertical line corresponding to the total points. (Figure3, Table 3)

Table 3

The results for each indicator in the Nomogram

\begin{tabular}{|llllll|}
\hline Indicators & Coefficient of prediction & Standard error & OR & $95 \% \mathrm{Cl}($ low-up) & $P$ \\
\hline Constant term & 6.19247 & 1.270 & 13.886 & $(1.153-167.182)$ & 0.038 \\
\hline Albumin & -0.03823 & 0.008 & 1.029 & $(1.012-1.046)$ & 0.001 \\
\hline Hypertension & 1.80654 & 0.382 & 4.578 & $(2.167-9.672)$ & $<0.001$ \\
\hline Diabetes & 1.82764 & 1.043 & 12.859 & $(1.666-99.245)$ & 0.014 \\
\hline FI & -0.28469 & 0.073 & 0.781 & $(0.678-0.901)$ & 0.001 \\
\hline VFI & -0.40586 & 0.083 & 0.776 & $(0.659-0.912)$ & 0.002 \\
\hline BUN & 0.19816 & 0.064 & 1.195 & $(1.054-1.354)$ & 0.006 \\
\hline
\end{tabular}

\section{Evaluation of the Nomogram model}

Internal test for this Nomogram using 1000 bootstrap methods showed a c-index of 0.896 ( $95 \% \mathrm{Cl}$ : $0.862-$ 0.930). The calibration curve showed that Nomogram had a good ability to predict the risk of chronic kidney disease. It was in good agreement with the actual risk of stage I-II chronic kidney disease diagnosed by KDOQI US[8]. Next, plot the Nomogram ROC curve for predicting the occurrence of chronic renal disease. The results were as follows: AUC0.884 (95\% Cl: $0.860-0.908)$; The optimal threshold is 0.663; Specificity $88.7 \%$; Sensitivity $78.0 \%$; Accuracy $82.0 \%$; The positive predictive value was $91.9 \%$. In conclusion, it could be seen that the histogram had a good effect on disease prediction.(Figure 4)

\section{DCA analysis of Nomogram}

Set the High Risk Threshold as the horizontal axis, and the Net Benefit as the vertical axis, and draw the DCA curve(Figure 5). As shown in the figure, the black curve (NONE) represented the net benefit of All individuals without CKD, the gray curve (ALL) represented the net benefit of All individuals with CKD, and 
the red curve (MODEL) represented the actual decision curve of All individuals in this study. The results showed that the Nomogram model had high application potential when the threshold probability exceeded 0.66 (the region between ALL and MODEL) for predicting CKD.

\section{Independent risk factor assessment for Nomogram}

In this study, ROC was tested and evaluated for the predictors BUN, albumin, FI and VFI. The results indicated that the best cutoff value of BUN was 5.9, Fi was 14.67, VFI was 4.6, and ALB was $40.26 \mathrm{~g} / \mathrm{L}$. (Figure 6, Table 4)

Table 4

ROC results of each independent risk factor indicator

\begin{tabular}{|lllllllll|}
\hline Predictors & AUC & $\begin{array}{l}\text { Standard } \\
\text { error }\end{array}$ & $\boldsymbol{Z}$ & $\boldsymbol{P}$ & $\begin{array}{l}\text { Youden } \\
\text { Index }\end{array}$ & $\begin{array}{l}\text { cut-off } \\
\text { value }\end{array}$ & sensitivity & specificity \\
\hline BUN & 0.691 & 0.019 & 10.058 & $<0.001$ & 0.295 & 5.9 & 52.20 & 77.33 \\
\hline FI & 0.694 & 0.019 & 10.488 & $<0.001$ & 0.308 & 14.67 & 50.80 & 80.00 \\
\hline VFI & 0.755 & 0.017 & 14.901 & $<0.001$ & 0.431 & 4.6 & 62.80 & 80.33 \\
\hline ALB & 0.590 & 0.021 & 4.350 & $<0.001$ & 0.181 & 40.26 & 85.40 & 32.67 \\
\hline
\end{tabular}

\section{Discussion}

CKD is defined as reduced kidney function or kidney damage from a variety of causes. CKD is divided into five stages based on glomerular filtration rate[10-11]. Renal function can also be measured by serum creatinine (SCR) or endogenous creatinine clearance [11]. Currently, ultrasound examination technology has been widely used in the prediction and diagnosis of different diseases. Xu et al [12] used real-time tissue elastography to predict the occurrence of esophageal varices in patients with chronic hepatitis $B$ associated cirrhosis. Simicic Majce et al [12] used contrast-enhanced ultrasound to assess the effect of renal reflux. De Freminville et al [15] predicted the mortality rate of renal transplant patients with renal resistance index $(\mathrm{RI})$ measured by ultrasound technique. Different from the clinical application of creatinine or glomerular filtration rate in the diagnosis of CKD, this study used color Doppler quantitative region of interest $(\mathrm{ROI})$ technique combined with assay indexes to predict the early onset of CKD and establish a Nomogram prediction model.

As previously reported [16], changes in renal blood perfusion are closely related to renal function. Unlike CTA (CT angiography) and radionuclide examination, color Doppler imaging provides a non-invasive, convenient, and non-radiological method for evaluating renal blood flow[17-18]. Color Doppler region of interest quantitative technology through the acquisition of original signals, combined with QLAB software quantitative analysis, calculate the renal blood perfusion index. VI represents the number of vascular beds in $\mathrm{ROI}$; Fi: represents the average flow rate of blood flow in all vessels in $\mathrm{ROI}$; $\mathrm{VFI}=\mathrm{VI} \times \mathrm{FI}$, then represents the blood perfusion of ROI[19-20]. At present, Liu [21] has applied the color Doppler region of interest quantitative technique to the prediction of intraoperative bleeding of scar uterine, and the results 
suggest that VI may be helpful in predicting intraoperative massive bleeding of scar uterine pregnancy. Mala et al [22] applies this technique to the diagnosis of polycystic ovary, and the results suggest that the adnadal VI and VFI of women with polycystic ovary are significantly increased, and the indicators measured by the quantitative region of interest (ROI) technique can be used as one of the diagnostic criteria for polycystic ovary. Therefore, this study is to investigate whether VI, FI and VFI could be used as diagnostic indicators of CKD.

In this study, it was found that the mean values of VI, FI and VFI in the CKD group were lower than those in the control group $(P<0.05)$, and FI and VFI were independent risk factors for early chronic kidney disease. Consistent with the results of D 'Amico et al[23], the number of vascular beds in the CKD group was reduced, the velocity of intravascular blood flow was slowed, and the perfusion of renal tissue was also reduced. Possible reasons for this result are: (1) Extracellular matrix deposition occurs during the development of chronic renal disease, resulting in interstitial fibrosis and other pathological changes. Subsequently, circulatory resistance increases, renal hemodynamics changes, and perfusion volume decreases. (2) After renal function is impaired, the activation of various cytokines leads to the increase of renal artery resistance, the decrease of intravascular velocity and the decrease of blood perfusion. (3) Under the state of oxidative stress, various signaling pathways are activated, leading to the decrease of renal blood perfusion. The above reasons together present the outcome of "high resistance and low perfusion", leading to the decrease of FI and VFI.

Multivariate logistic regression analysis suggested that albumin, hypertension, diabetes and urea nitrogen were also independent risk factors for stage I-II CKD. Albumin, as an indicator of nutritional status, is often used in the diagnosis of various chronic diseases. In patients with CKD, proteinuria occurs due to decreased glomerular filtration and loss of protein. Decrease in serum albumin, coupled with malnutrition in chronically ill patients and inadequate supplementation, leads to further albumin decline. According to ROC results, the truncation value of albumin was $40.26 \mathrm{~g} / \mathrm{L}$. When this value is exceeded, physicians should be on guard against the occurrence of chronic kidney disease in combination with the medical history. When patients develop hypertension, renal hemodynamics will change. Persistent hyperfiltration leads to impaired glomerular barrier function, which in turn leads to limited glomerular filtration. The organ most commonly affected by diabetes is the kidney. The damage of high glucose to the kidneys involves not only the glomeruli but also the tubules. Hypertension and diabetes mellitus are risk factors for CKD progression to end-stage renal disease [24-25]. Therefore, early and effective control of blood pressure and blood glucose can prevent the occurrence and deterioration of CKD to a certain extent. According to the histogram, it can be seen that BUN also has a good ability to identify CKD in the early stage. Urea nitrogen is filtered out of the glomerulus. When microcirculation is damaged and basement membrane is damaged, BUN will increase inversely proportional to the glomerular filtration rate [26]. In summary, FI, VFI, BUN, albumin, hypertension and diabetes are independent risk factors for early CKD, and Nomogram can provide an effective risk assessment for CKD.

In comparison of baseline data, there was no statistically significant difference in RI between the CKD group and the control group. It may be because in the early stage of CKD, although the renal blood 
perfusion is reduced, the renal vasoconstriction is not obvious. In the early stage, the kidney is in the compensatory stage, and there are few structural changes, such as renal length and cortical thickness. This findings suggest that gender has no role in early CKD, but previous studies have found a 9 percent higher prevalence in women than in men [27]. Gender and age did not become independent risk factors in this study. The possible reason is that the included samples are relatively limited and biased. The scope of the included population can be appropriately expanded, the sample size can be appropriately increased, and more patients from different regions can be included in the study. The weakness of this study is the lack of external verification for Nomogram, which requires a large amount of data to make prospective prediction.

\section{Conclusion}

The color Doppler region of interest (ROI) quantitative technique was applied to the prediction of CKDI-II stage in this study. The C-index of this Nomogram was 0.896 , which indicated that this Nomogram had good discriminative power. The Nomogram model suggested a threshold of 0.663 . The risk $>0.663$ of patients with CKD is considered as a high-risk group, and intervention is recommended as soon as possible. The construction of this Nomogram model has a good indication for sonographers. It has certain reference significance for the early detection of CKD, and can give timely feedback to clinicians.

\section{Declarations}

\section{Ethical Approval and Consent to participate}

All procedures performed in studies involving human participants were in accordance with the ethical standards of the institutional and/or national research committee and with the 1964 Helsinki Declaration and its later amendments or comparable ethical standards. The study was approved by the Bioethics Committee of the Affiliated Hospital of Qingdao University (QYFYWZLL26280).

\section{Consent for publication}

Informed consent was obtained from all individual participants included in the study.

The authors affirm that human research participants provided informed consent for publication of the images in Figure1.

Patients signed informed consent regarding publishing their data and photographs.

\section{Availability of data and materials}

Data and materials in the article are available.

\section{Competing interests}


The authors have no relevant financial or non-financial interests to disclose.

The authors have no competing interests to declare that are relevant to the content of this article.

All authors certify that they have no affiliations with or involvement in any organization or entity with any financial interest or non-financial interest in the subject matter or materials discussed in this manuscript.

The authors have no financial or proprietary interests in any material discussed in this article.

\section{Funding}

Not applicable

\section{Authors' contributions}

(I) Conception and design: L Zhang; KS Xu.

(II) Administrative support: KS Xu.

(III) Provision of study materials or patients: LY Ma; CJ Shao; Kuishuai Xu.

(IV) Collection and assembly of data: L Zhang;LY Ma.

(V) Data analysis and interpretation: L Zhang; KS Xu.

(VI) Manuscript writing: All authors

(VII) Final approval of manuscript: All authors

\section{Acknowledgements}

Not applicable

\section{Authors' information}

Liang Zhang ${ }^{1 \#, ~ K u i s h u a i ~} \mathrm{Xu}^{2 \#^{*}}$, Leiyuan $\mathrm{Ma}^{1}$, Changjie Shao ${ }^{1}$

1 Department of Abdonminal Ultrasound, The Affiliated Hospital of Qingdao University, Qingdao 266000, Shandong, China

2 Department of Sports Medicine, The Affiliated Hospital of Qingdao University, Qingdao 266000, Shandong, China

\#These authors Liang Zhang and Kuishuai Xu contributed equally.

Corresponding author: *Kuishuai Xu, The Affiliated Hospital of Qingdao University, 26600, Email:1213218702@qq.com phone numbers: 0086+0532+17852025589

\section{References}

Citation list not provided with this version 


\section{Figures}

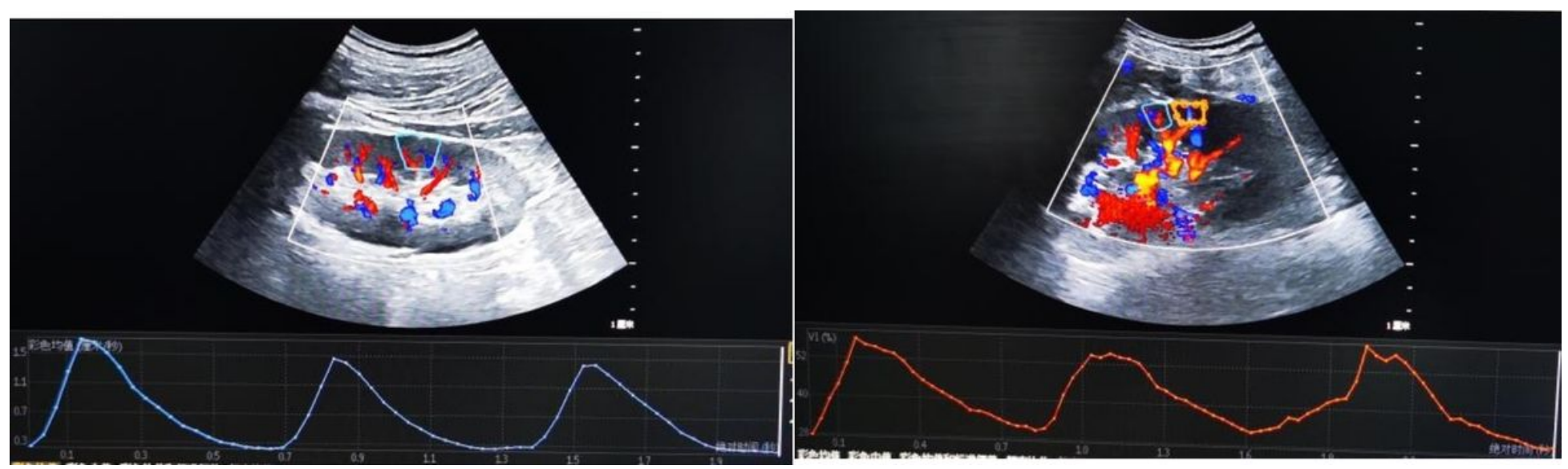

\section{Figure 1}

Images taken by color Doppler ultrasound The color Doppler area of interest diagram of the control group is on the left; Color Doppler area of interest image of the experimental group on the right. 
500 patients with chronic kidney disease and 300 in the control group were included according to the criteria.

Based on one-way ANOVA, meaningful baseline indicators were calculated.

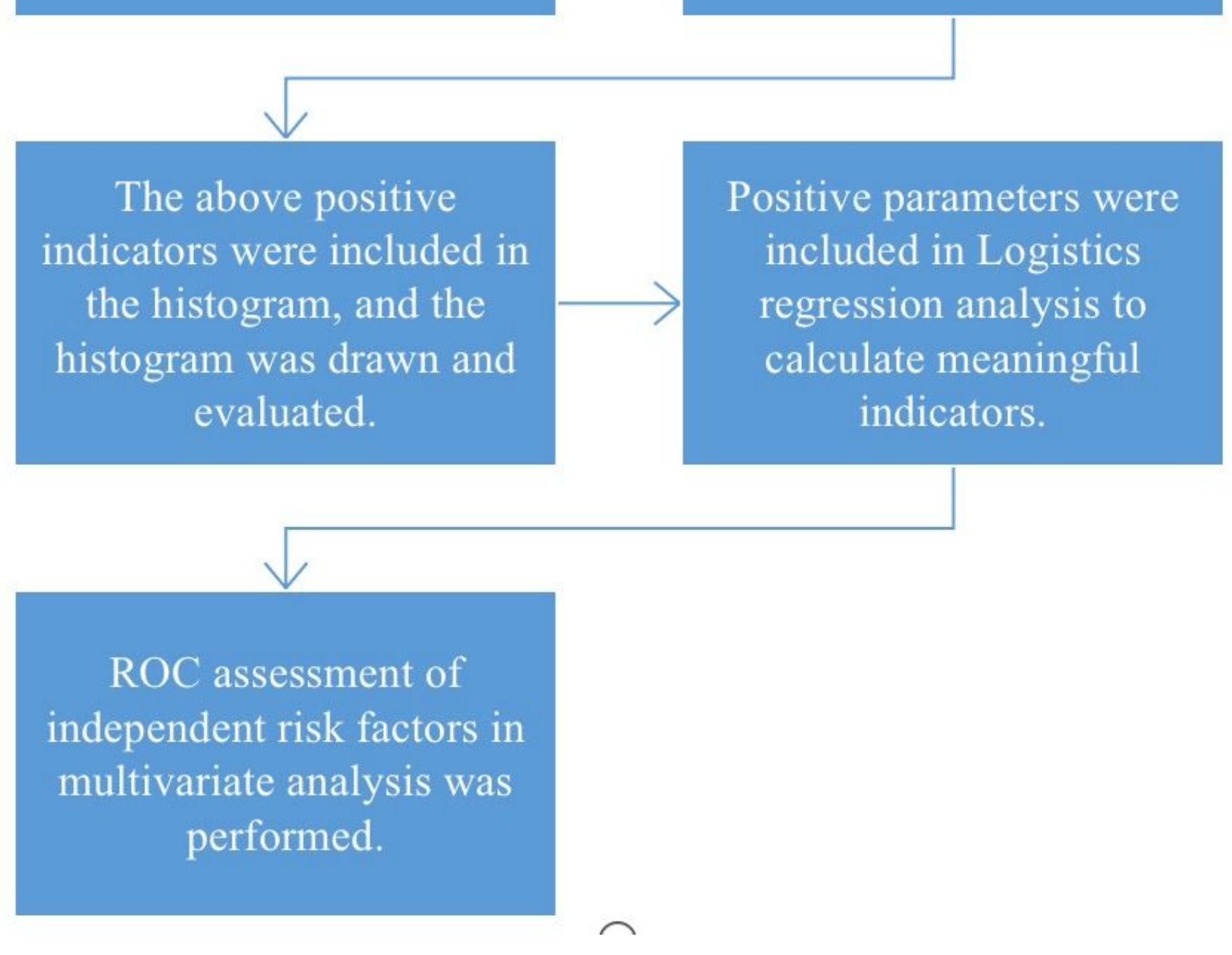

\section{Figure 2}

Flow of participants 
$B P$

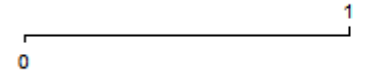

$\mathrm{FI}$

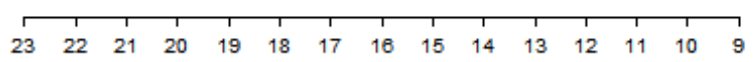

DM

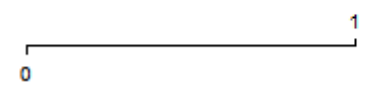

BUN

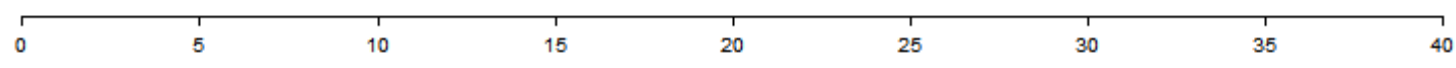

VFI

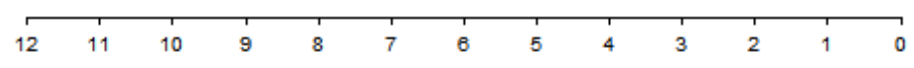

ALB

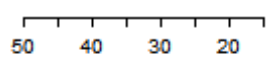

Total Points

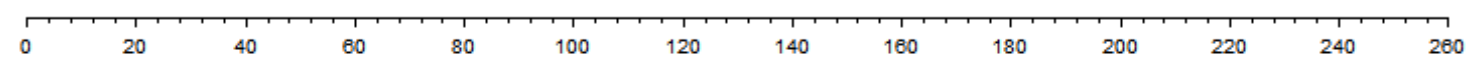

Linear Predictor

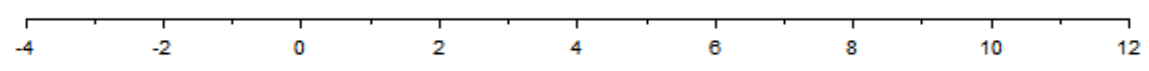

CKD

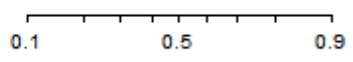

\section{Figure 3}

Nomogram model for predicting the occurrence of CKD BP is hypertension, 1: positive, 0 : negative; DM is diabetic, 1: positive, 0 : negative. 

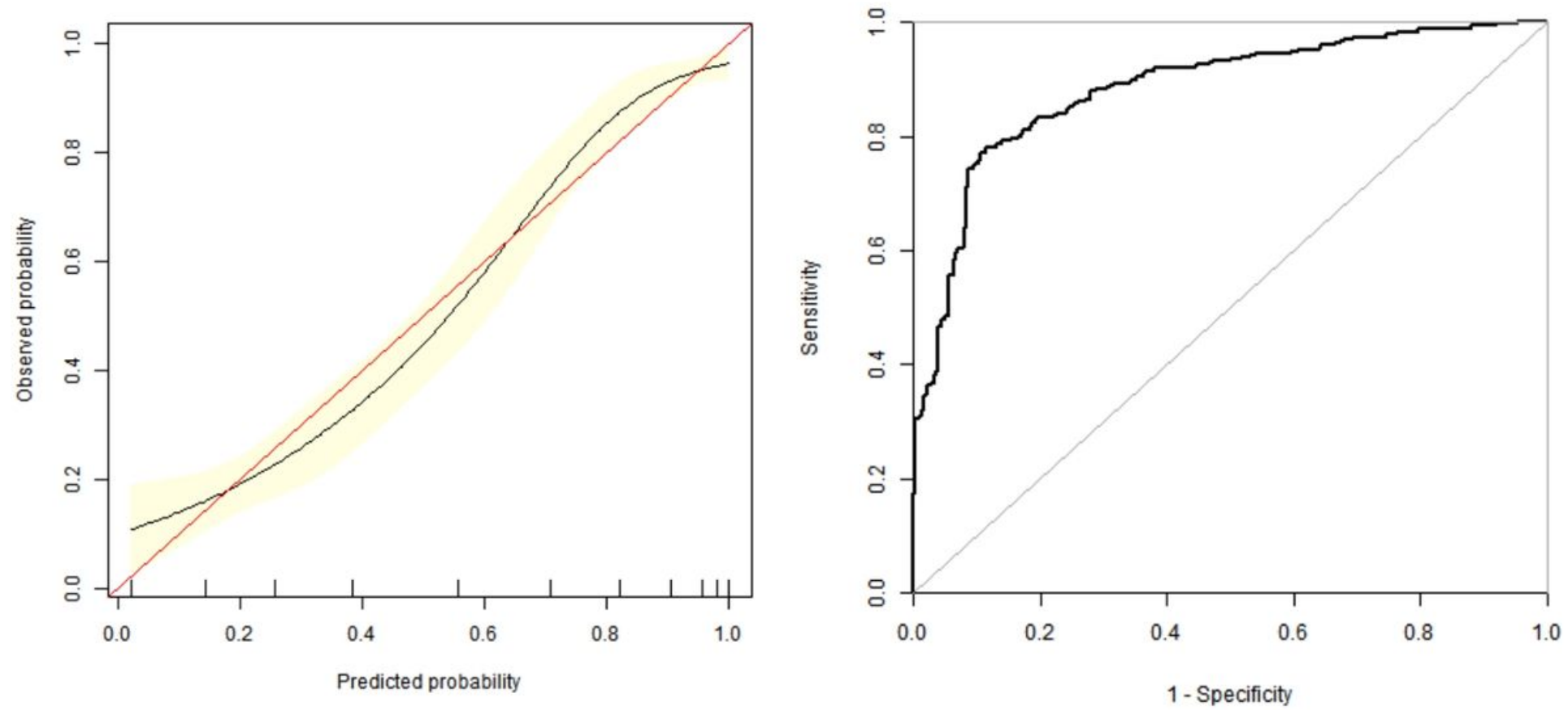

Figure 4

Calibration curve and ROC curve of Nomogram On the left is the calibration curve; On the right is the ROC curve of Nomogram. 


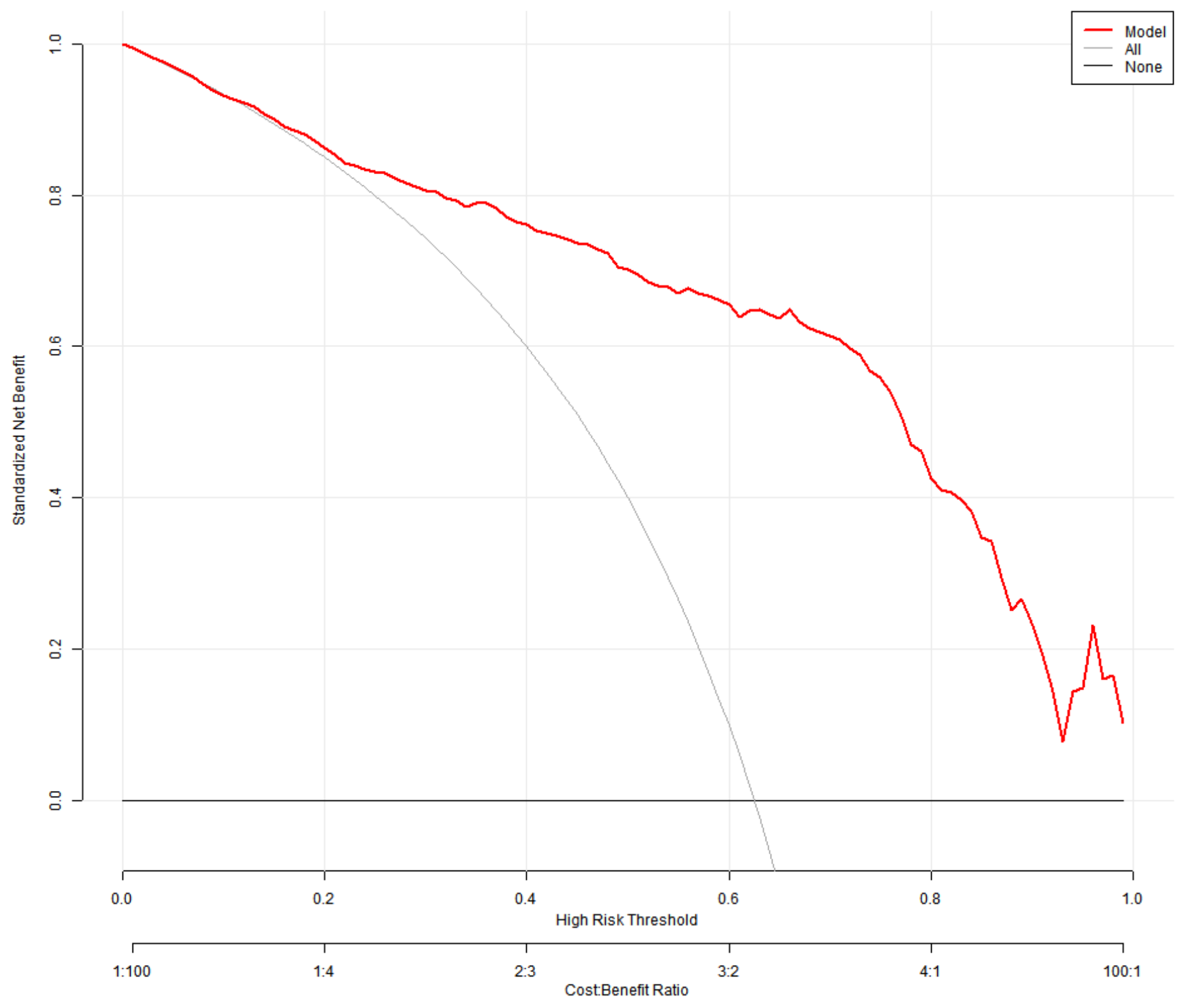

Figure 5

the DCA curve of Nonogram 


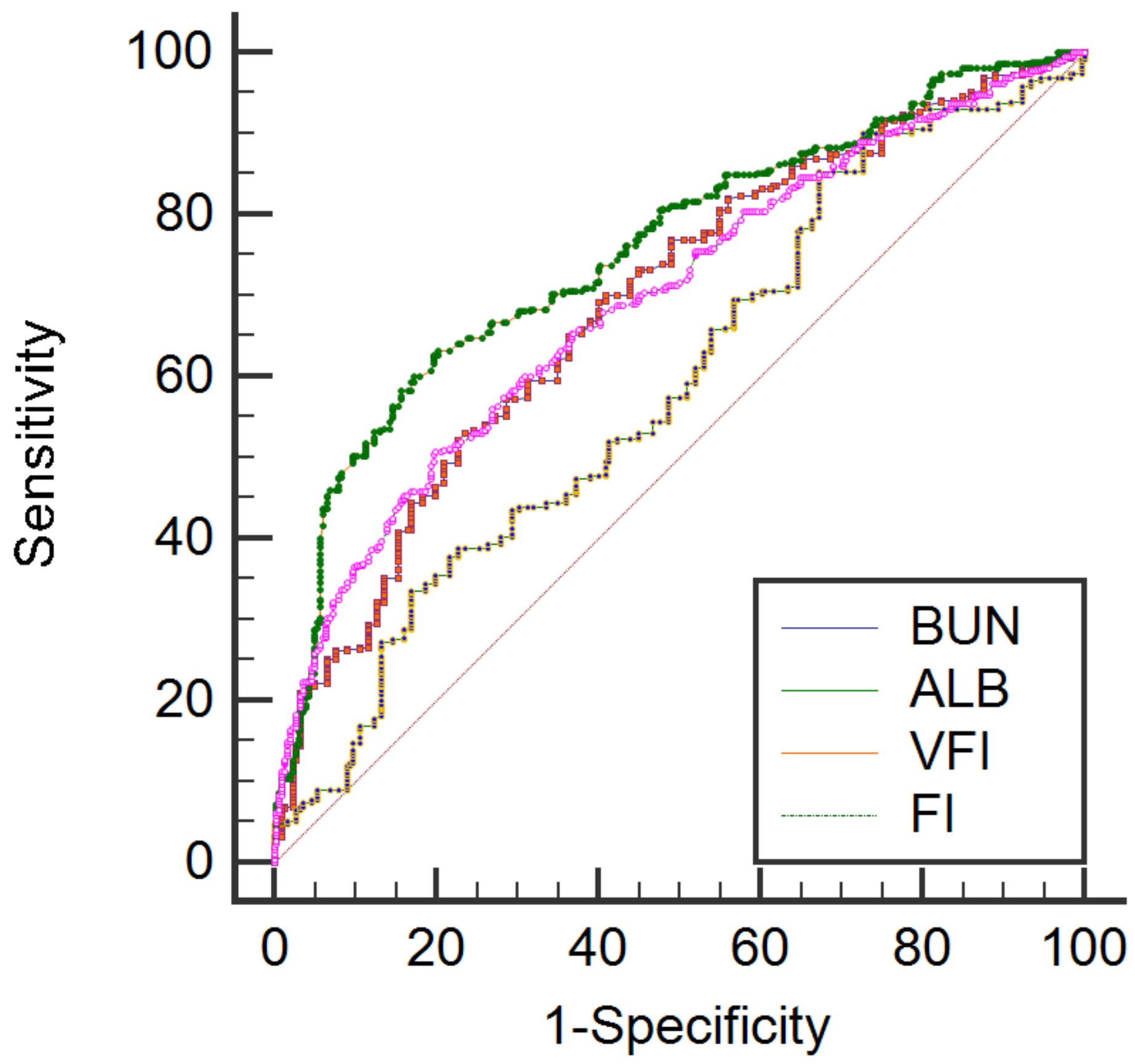

Figure 6

ROC curve of independent risk factors. 